\title{
Budd-Chiari Syndrome During Long-term Follow-up after Allogeneic Umbilical Cord Blood Transplantation
}

\author{
Yusuke Akitomi ${ }^{1}$, Yoshinari Asaoka ${ }^{1}$, Ryo Miura ${ }^{1}$, Sae Murata ${ }^{1}$, Takatsugu Yamamoto ${ }^{1}$, \\ Haruko Tashiro ${ }^{1}$, Naoki Shirafuji ${ }^{1}$, Kentaro Yamada $^{2}$, Masayoshi Yamamoto ${ }^{2}$, Hiroshi Kondo ${ }^{2}$ \\ and Atsushi Tanaka ${ }^{1}$
}

\begin{abstract}
:
A series of abdominal computed tomography scans of an asymptomatic 40-year-old woman with a history of umbilical cord blood transplantation (CBT) for leukemia at 19 years old revealed the long-term gradual development of a right hepatic vein thrombus and stenosis of the inferior vena cava, leading to a diagnosis of Budd-Chiari syndrome. The Budd-Chiari syndrome in this case might have been influenced by the patient's history of multiple liver abscesses after CBT and associated thrombus formation, in addition to the hormone replacement therapy with estradiol and dydrogesterone she was taking. This case provides insight into the development of Budd-Chiari syndrome.
\end{abstract}

Key words: Acute lymphoblastic leukemia, Allogeneic umbilical cord blood transplantation, Budd-Chiari syndrome, Liver abscess

(Intern Med Advance Publication)

(DOI: 10.2169/internalmedicine.8226-21)

\section{Introduction}

Budd-Chiari syndrome is a very rare disorder characterized by obstruction or stenosis of the main hepatic vein or hepatic portion of the inferior vena cava (IVC) that results in hepatic dysfunction and portal hypertension, which has a prevalence of 3.2 per million in the population of Japan (1). The chronic form with gradual portal hypertension is more common in Asia, whereas the acute form is more common in Europe and the USA, with hepatomegaly and ascites due to acute obstruction or stenosis, leading to death from liver failure.

The etiology of primary Budd-Chiari syndrome is unclear, but it has been suggested to involve thrombophilia, myeloproliferative disorders, and oral contraceptive use (2). To our knowledge, there have been no case reports of BuddChiari syndrome documenting the course of stenosis in the hepatic portion of the IVC over a long period via imaging studies.
We herein report a case of Budd-Chiari syndrome that developed gradually without treatment for more than 15 years, which we were able to examine using contrast-enhanced computed tomography (CT), providing insight into the pathogenesis of the syndrome.

\section{Case Report}

A 40-year-old Japanese woman presented for follow-up imaging examinations of multiple microcalcifications in the liver and liver deformation. She had developed acute lymphoblastic leukemia (ALL) at 19 years old, and after achieving remission via chemotherapy, she underwent allogeneic umbilical cord blood transplantation (CBT) with myeloablative conditioning including total body irradiation (12 Gy). After transplantation, she developed acute graft-versus-host disease (GVHD) (gastrointestinal tract stage 4, overall grade IV) and was treated with high-dose steroids for a long time. Multiple microabscesses were found in her liver, and antibiotic treatment was successful. The microabscesses turned

${ }^{1}$ Department of Medicine, Teikyo University School of Medicine, Japan. and ${ }^{2}$ Department of Radiology, Teikyo University School of Medicine, Japan.

Received: June 26, 2021; Accepted: September 1, 2021; Advance Publication by J-STAGE: October 26, 2021

Correspondence to Dr. Yoshinari Asaoka, y-asaoka@med.teikyo-u.ac.jp 
Table. Laboratory Test Results.

\begin{tabular}{|c|c|}
\hline WBC & $7,400 / \mu \mathrm{L}$ \\
\hline Neutrophils & $68 \%$ \\
\hline Lymphocytes & $23 \%$ \\
\hline Monocytes & $8 \%$ \\
\hline $\mathrm{RBC}$ & $380 \times 10^{4} / \mu \mathrm{L}$ \\
\hline $\mathrm{Hb}$ & $11.0 \mathrm{~g} / \mathrm{dL}$ \\
\hline Hct & $34.3 \%$ \\
\hline PLT & $21.0 \times 10^{4} / \mu \mathrm{L}$ \\
\hline PT & $10.5 \mathrm{~s}$ \\
\hline PT activity & $100 \%$ \\
\hline PT-INR & 0.93 \\
\hline APTT & $27.0 \mathrm{~s}$ \\
\hline FIB & $371 \mathrm{mg} / \mathrm{dL}$ \\
\hline TP & $7.6 \mathrm{~g} / \mathrm{dL}$ \\
\hline Alb & $4.2 \mathrm{~g} / \mathrm{dL}$ \\
\hline T-Bil & $0.61 \mathrm{mg} / \mathrm{dL}$ \\
\hline D-Bil & $0.04 \mathrm{mg} / \mathrm{dL}$ \\
\hline I-Bil & $0.57 \mathrm{mg} / \mathrm{dL}$ \\
\hline AST & $23 \mathrm{U} / \mathrm{L}$ \\
\hline ALT & $19 \mathrm{U} / \mathrm{L}$ \\
\hline LDH & $186 \mathrm{U} / \mathrm{L}$ \\
\hline ALP & $152 \mathrm{U} / \mathrm{L}$ \\
\hline$\gamma$-GTP & $45 \mathrm{U} / \mathrm{L}$ \\
\hline BUN & $13.9 \mathrm{mg} / \mathrm{dL}$ \\
\hline Cre & $0.51 \mathrm{mg} / \mathrm{dL}$ \\
\hline UA & $5.2 \mathrm{mg} / \mathrm{dL}$ \\
\hline $\mathrm{Na}$ & $141 \mathrm{mEq} / \mathrm{L}$ \\
\hline $\mathrm{Cl}$ & $100 \mathrm{mEq} / \mathrm{L}$ \\
\hline $\mathrm{K}$ & $3.9 \mathrm{mEq} / \mathrm{L}$ \\
\hline NH3 & $44 \mu \mathrm{g} / \mathrm{dL}$ \\
\hline Glu & $103 \mathrm{mg} / \mathrm{dL}$ \\
\hline CRP & $0.55 \mathrm{mg} / \mathrm{dL}$ \\
\hline Syphilis RPR & $(-)$ \\
\hline HBs Ag & $(-)$ \\
\hline $\mathrm{HCV} \mathrm{Ab}$ & $(-)$ \\
\hline \multicolumn{2}{|c|}{$\begin{array}{l}\text { Alb: albumin, ALP: alkaline phosphatase, ALT: } \\
\text { alanine aminotransferase, APTT: activated par- } \\
\text { tial thromboplastin time, AST: aspartate amino- } \\
\text { transferase, BUN: blood urea nitrogen, Cre: } \\
\text { creatinine, CRP: C-reactive protein, D-Bil: di- } \\
\text { rect bilirubin, FIB: fibrinogen, Glu: glucose, } \\
\text { Hb: hemoglobin, HBs Ag: hepatitis B surface } \\
\text { antigen, Hct: hematocrit, HCV Ab: hepatitis C } \\
\text { virus antibody, I-Bil: indirect bilirubin, LDH: } \\
\text { lactate dehydrogenase, PLT: platelet, PT: pro- } \\
\text { thrombin time, PT-INR: prothrombin time-in- } \\
\text { ternational normalized ratio, RBC: red blood } \\
\text { cell, RPR: rapid plasma reagin, T-Bil: total bili- } \\
\text { rubin, TP: total protein, UA: uric acid, WBC: } \\
\text { white blood cell, } \gamma \text {-GTP: } \gamma \text {-glutamyl transferase }\end{array}$} \\
\hline
\end{tabular}

into calcifications measuring about $3 \mathrm{~mm}$. No abnormalities, such as inflammatory reactions, were observed, and she was followed up regularly with imaging examinations on an outpatient basis.

At 20 years after the onset of ALL, she underwent follow-up abdominal plain CT, which revealed suspected stenosis of the hepatic portion of the IVC. Therefore, she was referred to the Department of Gastroenterology, although she had no subjective symptoms.

Her medical history included luteal insufficiency after umbilical CBT since 20 years old, for which she has been on hormone replacement therapy (HRT) with estradiol and dydrogesterone from 20 to 40 years old. She also underwent laparoscopic bilateral salpingo-oophorectomy for bilateral ovarian cysts at 35 years old. She had no history of drinking alcohol or smoking. Her family history was unremarkable.

The patient's vital signs were stable, and a physical examination revealed mildly dilated subcutaneous veins running longitudinally in her abdomen, mild pitting edema, and mild capillary dilation in the lower legs. Laboratory test results were generally normal (Table).

Abdominal ultrasound showed dullness of the liver edge, congestion of the middle hepatic vein, and collapse of the right hepatic vein (Fig. 1). Abdominal contrast-enhanced CT showed multiple microcalcifications in the liver, stenosis of the hepatic portion of the IVC, dilatation of the left hepatic vein and middle hepatic vein, and the development of collateral veins, mainly the azygos venous system (Fig. 2). We checked previous images for the lesion responsible for stenosis of the hepatic portion of the IVC and found that the right hepatic vein had been open on abdominal contrastenhanced CT performed 14 years earlier (Fig. 3A, B). Abdominal contrast-enhanced CT performed 11 years earlier showed microcalcifications along the right hepatic vein and thrombotic occlusion in the same area, which was continuous with the hepatic portion of the IVC, indicating severe stenosis of the hepatic portion of the IVC (Fig. 3C, D, E, F). Esophagogastroduodenoscopy revealed gastric varices, classified as $\mathrm{Lg}-\mathrm{b}, \mathrm{F} 2, \mathrm{Cw}$, and $\mathrm{RC} 0$ according to the Japan Society for Portal Hypertension classification (Fig. 4). Based on the imaging findings, we made a diagnosis of Budd-Chiari syndrome.

This case was indicated for treatment because the patient was young, and her condition was associated with a decline in the hepatic reserve and risk of exacerbation of gastric varices. We decided to use percutaneous transluminal angioplasty (PTA) to release the stenosis of the hepatic portion of the IVC.

First, we performed angiography of the common trunk of the left hepatic vein and the middle hepatic vein via a transjugular approach and observed stenosis at the confluence of the IVC with the common trunk of the left hepatic vein and the middle hepatic vein (Fig. 5A), which had not been present on prior abdominal contrast-enhanced CT. As the mean venous pressure in the right atrium was $0 \mathrm{mmHg}$ and that in the common duct of the left hepatic vein and middle hepatic vein was $10 \mathrm{mmHg}$, PTA was performed with a balloon $12 \mathrm{~mm}$ in diameter in this area where there was a pressure difference. Angiography was then performed from the caudal side of the stenosis in the IVC and showed severe stenosis at the hepatic portion of the IVC and marked dilatation of the azygos venous system as a development of collateral veins (Fig. 5B). As the mean venous pressure in 
A

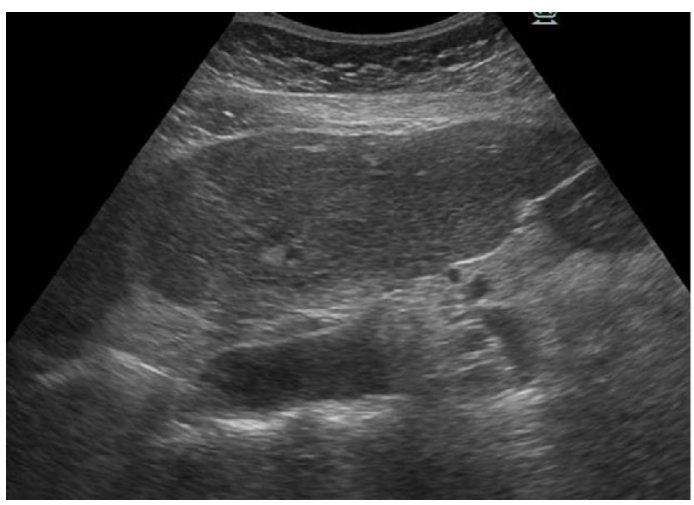

B

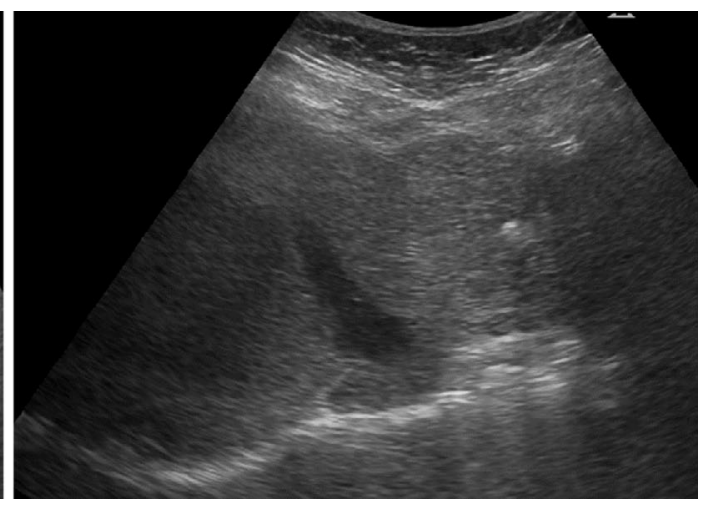

Figure 1. Abdominal ultrasound showed dullness of the liver edge (A), congestion in the middle hepatic vein, and collapse of the right hepatic vein (B).
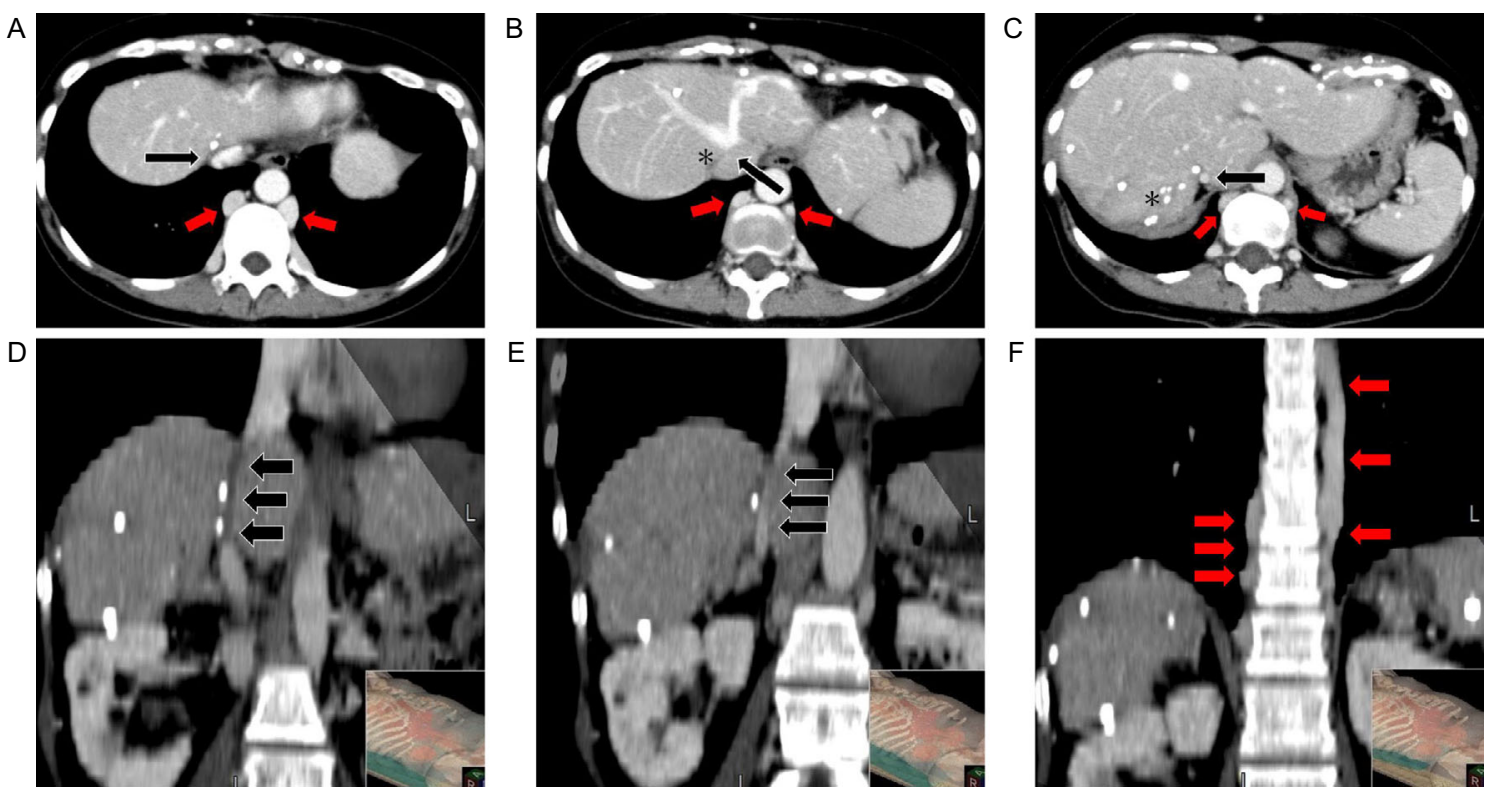

Figure 2. Abdominal contrast-enhanced CT showed multiple microcalcifications in the liver, stenosis of the hepatic portion of the IVC (black arrows in A-E), obstruction in the right hepatic vein (asterisks in $B, C$ ), dilatation of the left hepatic vein and middle hepatic vein, and the development of collateral blood vessels, mainly the azygos venous system (red arrows in A, B, C, F).

the right atrium was $0 \mathrm{mmHg}$ and that in the hepatic portion of the IVC was $13 \mathrm{mmHg}$, PTA was also performed with a balloon $12 \mathrm{~mm}$ in diameter in this area where there was a pressure difference. Finally, the mean venous pressure in the right atrium was $6 \mathrm{mmHg}$, that in the common duct of the left hepatic vein and middle hepatic vein was $4 \mathrm{mmHg}$, that in the hepatic portion of the IVC was $14 \mathrm{mmHg}$, and PTA was completed, as improvement of stenosis in the hepatic portion of the IVC was confirmed by digital subtraction angiography (DSA) (Fig. 5C).

There were no complications from PTA. Clopidogrel and aspirin were started on the day after PTA to prevent thrombotic occlusion, and HRT was discontinued due to its potential to induce Budd-Chiari syndrome. No new subjective symptoms appeared after PTA, and the patient was discharged seven days later and followed up on an outpatient basis.

At three months after PTA, follow-up DSA showed restenosis in the hepatic portion of the IVC, and retreatment was planned (Fig. 5D).

\section{Discussion}

Budd-Chiari syndrome is a very rare disorder that presents with hepatic dysfunction and portal hypertension due to obstruction or stenosis of the main hepatic vein or hepatic portion of the IVC. The etiology of primary Budd-Chiari syndrome is still unclear, but it has been suggested to involve thrombophilia, myeloproliferative disorders, and the use of oral contraceptives (2). It can be differentiated from veno-occlusive disease/sinusoidal obstruction syndrome (VOD/SOS), a known complication of hematopoietic stem 

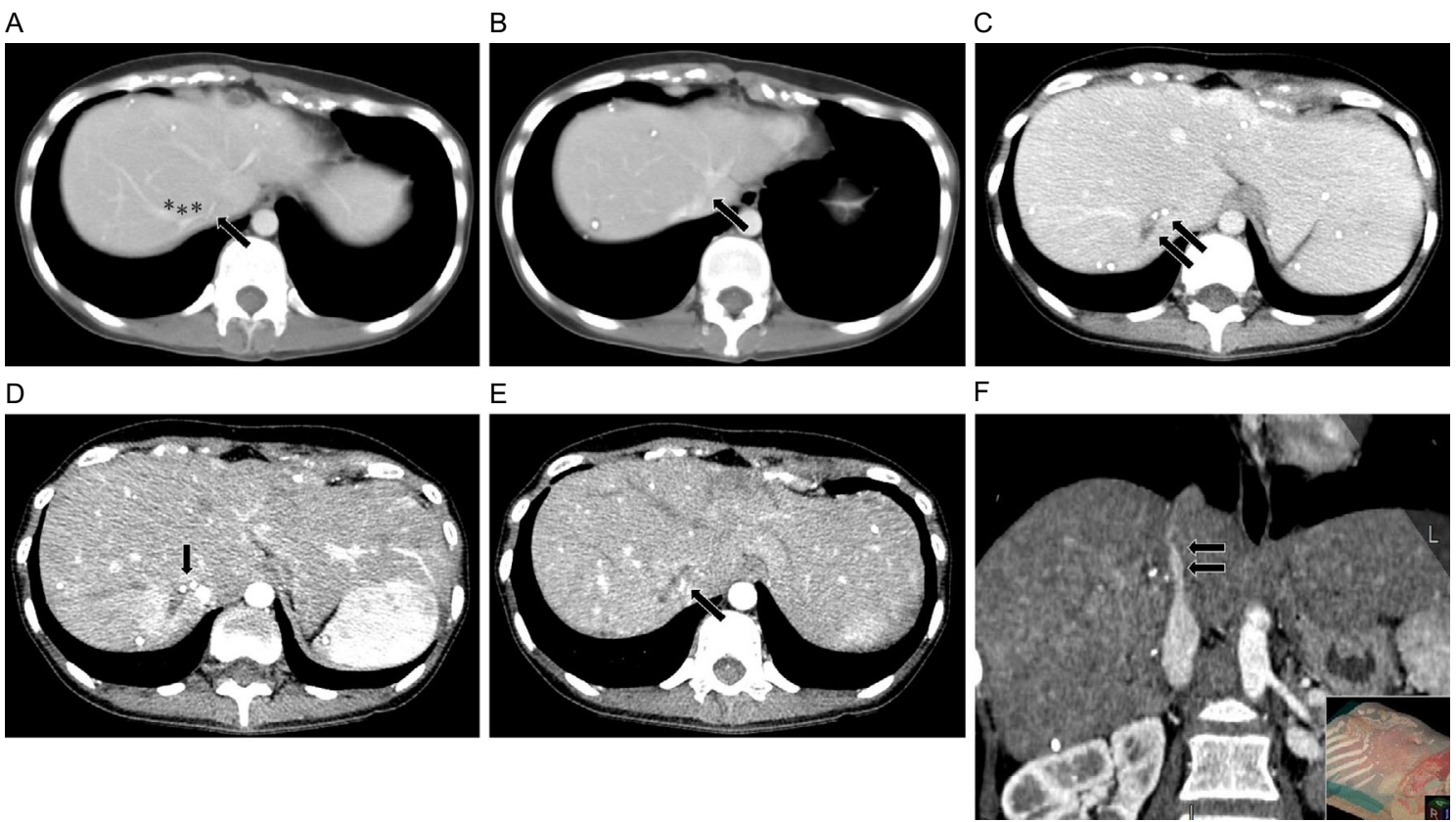

Figure 3. Abdominal contrast-enhanced CT performed 14 years earlier showed no significant abnormalities in the IVC other than physiological mild stenosis associated with flexion of the IVC (arrows in $\mathrm{A}$ and $\mathrm{B}$ ), and the right hepatic vein had been open (asterisks in A). Abdominal contrast-enhanced CT performed 11 years earlier showed microcalcifications along the right hepatic vein and thrombotic occlusion in the same area (arrows in $\mathrm{C}$, D), which was continuous with the hepatic portion of the IVC, with severe stenosis of the hepatic portion of the IVC observed with contrast imaging (arrow in E, F).

A

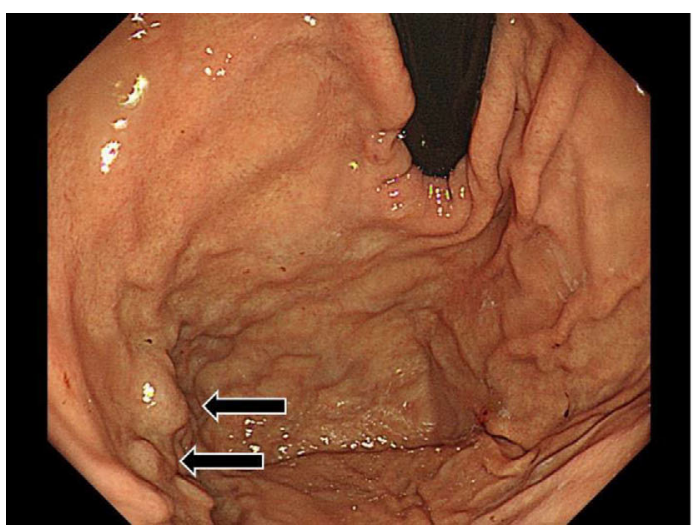

B

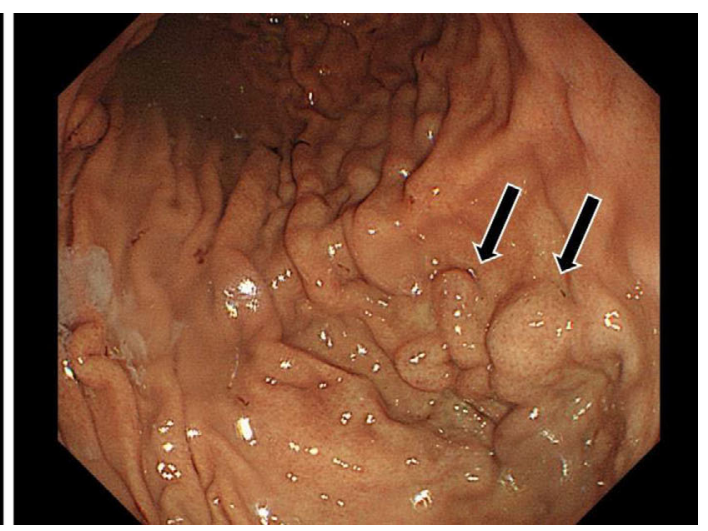

Figure 4. Esophagogastroduodenoscopy revealed gastric varices (arrows), classified as Lg-b, F2, Cw, and RC0 according to the Japan Society for Portal Hypertension classification (A, B).

cell transplantation (HSCT) and chemotherapy, as the occlusion in VOD/SOS occurs in a sinusoid or central vein (3). The pathogenesis of liver-specific damage other than chronic GVHD and VOD/SOS during long-term remission after HSCT is largely unknown and has been discussed only rarely in the literature (4).

In addition to HRT with estradiol and dydrogesterone, the Budd-Chiari syndrome in this case was suspected to be due to leukemia and HSCT used for its treatment. However, most cases of primary Budd-Chiari syndrome associated with leukemia are due to thrombosis associated with dis- seminated intravascular coagulopathy and rarely to intravenous infiltration of fungi from liver abscesses $(5,6)$ or gemtuzumab ozogamicin (7). All of these conditions were considered to be different from the pathogenesis in this case, as they occur within a short period of time after the onset of leukemia and have an acute course. In addition, no cases of Budd-Chiari syndrome during long-term remission after HSCT have been reported.

Other possible causes of Budd-Chiari syndrome in this case were suspected to be calcifications after liver abscesses (8) and liver deformation. These causes often lead to 
A

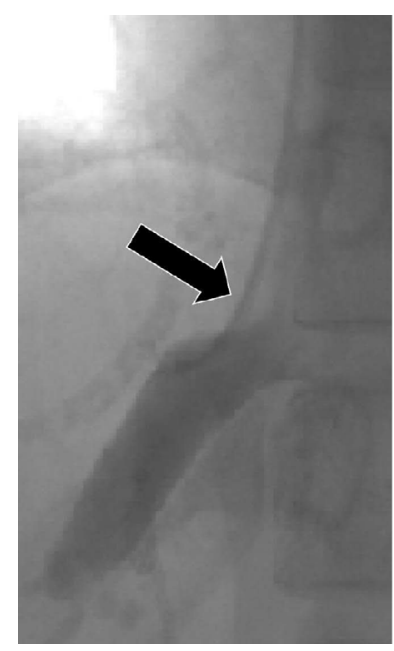

B

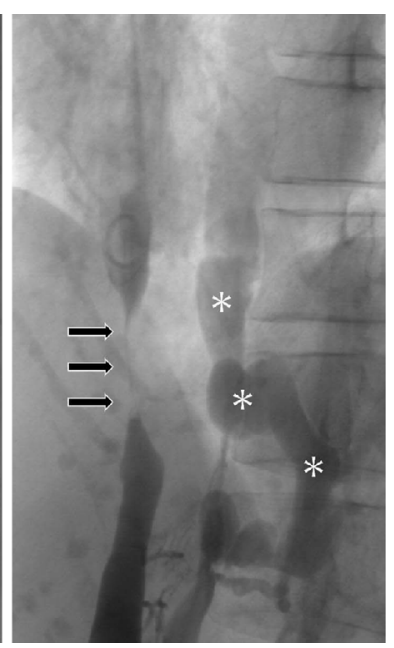

C

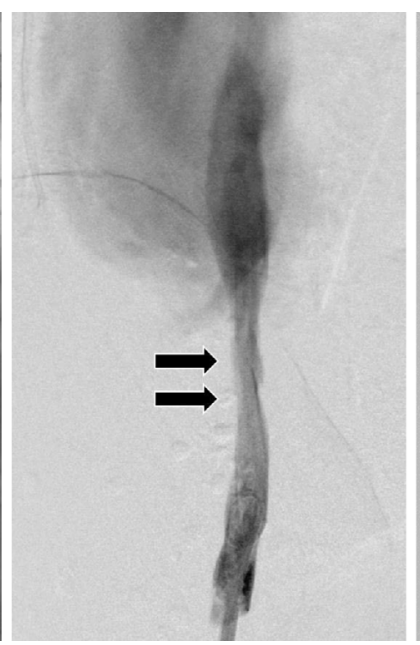

D

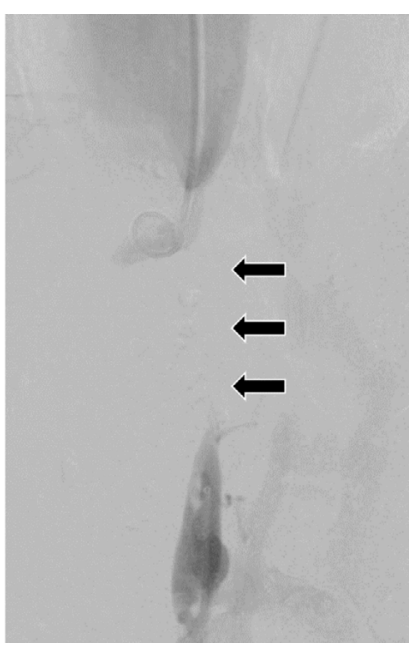

Figure 5. Angiography (anteroposterior view) showed stenosis at the confluence of the IVC and the common trunk of the left hepatic vein and middle hepatic vein (arrow in A). Angiography (lateral anterior oblique view) showed severe stenosis of the hepatic portion of the IVC (arrows in B) and marked dilatation of the azygos venous system as a development of collateral veins (asterisks in B). DSA (anteroposterior view) after PTA showed improvement of stenosis of the hepatic portion of the IVC (arrows in C), but restenosis was observed by follow-up study after three months (arrows in D).

the development of secondary Budd-Chiari syndrome due to compression of the IVC by large structures exhibiting calcification or liver deformation (especially enlargement of the caudate lobe) (9-13), and they were ruled out in this case because the calcifications were small and there was no clear compression of the IVC due to liver deformation. As the patient had a history of multiple liver abscesses, the possibility of hepatic vena cava syndrome (HVCS) was also considered, although this is rare outside developing countries. HVCS is a disease in which the main lesion arises from stenosis and occlusion after healing of focal thrombophlebitis at the confluence of the hepatic vein and the IVC due to bacteremia associated with poor hygiene (9, 14-16).

In this case, the patient had been followed up with imaging examinations for a long time after HSCT. Abdominal contrast-enhanced CT performed 14 years earlier showed multiple calcifications around the hepatic veins, but the right hepatic vein had still been open (Fig. 3A, B). Abdominal contrast-enhanced CT performed 11 years earlier showed progression of thrombotic occlusion of the right hepatic vein with internal calcifications (Fig. 3C, D). The progression of this thrombotic occlusion resulted in severe stenosis at the hepatic portion of the IVC (Fig. 3E, F). Thus, the most likely cause of Budd-Chiari syndrome in this case was thrombus formation in the right hepatic vein and its extension into the IVC. Although leukemia, umbilical CBT, associated complications, and HRT with estradiol and dydrogesterone were thought to be involved in the development of this disease, the impacts of these factors on thrombus formation are unclear.

The gastric varices in this case are detected in the gastric body (Fig. 4). Gastric body varices are rare, and BuddChiari syndrome may be related to the pathogenesis, but to our knowledge, there have been no reports suggesting BuddChiari syndrome as a background disease for varices in the gastric body. In our speculation, the splenic-renal shunt may flow in the opposite direction to the flow seen in liver cirrhosis, and this may be involved in the development of gastric body varices in this case. As the flows of the left and middle hepatic veins to the right atrium were kept intact, although the right hepatic vein and hepatic IVC were stenotic, collateral flow developed through the left renal vein, gastric varices, splenic vein, and portal vein to the hepatic left lobe. In addition, compensatory hypertrophy of the left lobe may have contributed to the preservation of the liver function, although hepatic dysfunction occurs in most patients with Budd-Chiari syndrome with a long-term course.

In this case, PTA, which is the first-line treatment for stenosis of the hepatic portion of the IVC due to its high efficacy and safety, was performed (3, 17-19). Restenosis was observed at three months after PTA (Fig. 5D). As mild gastric varices and elevated liver enzyme levels were found due to the lack of treatment for a long period, it was thought that large-diameter balloon dilatation or stenting should be attempted as the next treatment, considering the young age of the patient (18).

\section{Conclusion}

We encountered a case of Budd-Chiari syndrome during long-term remission after allogeneic umbilical CBT for ALL. Abdominal contrast-enhanced CT performed 11 years earlier had suggested thrombus formation in the right hepatic vein associated with multiple microcalcifications in the liver and extension to the IVC. The pathogenesis of liverspecific damage other than chronic GVHD and VOD/SOS during long-term remission after HSCT is largely unknown. 
With advances in therapies, such as HSCT and antibiotics, the number of patients under long-term follow-up after leukemia and treatment-related infectious diseases have been completely cured is increasing. This case suggests that cured multiple liver abscesses after leukemia treatment may lead to the gradual development of hepatic vein thrombosis and Budd-Chiari syndrome.

The English in this document has been checked by at least two professional editors, both native speakers of English. For a certificate, please see: http://www.textcheck.com/certificate/HBR Dnw

Grant Support: This work was supported by a Health and Labor Sciences Research Grant (research on intractable hepatobiliary disease) issued by the Ministry of Health, Labor, and Welfare of Japan.

The authors state that they have no Conflict of Interest (COI).

\section{References}

1. Ohfuji S, Furuichi Y, Akahoshi T, et al. Japanese periodical nationwide epidemiologic survey of aberrant portal hemodynamics. Hepatol Res 49: 890-901, 2019.

2. Darwish Murad S, Plessier A, Hernandez-Guerra M, et al. Etiology, management, and outcome of the Budd-Chiari syndrome. Ann Intern Med 151: 167-175, 2009.

3. Valla DC. Budd-Chiari syndrome and veno-occlusive disease/sinusoidal obstruction syndrome. Gut 57: 1469-1478, 2008.

4. Majhail NS. Long-term complications after hematopoietic cell transplantation. Hematol Oncol Stem Cell Ther 10: 220-227, 2017.

5. Young RC. The Budd-Chiari syndrome caused by Aspergillus; two patients with vascular invasion of the hepatic veins. Arch Intern Med 124: 754-757, 1969.

6. Vallaeys JH, Praet MM, Roels HJ, Van Marck E, Kaufman L. The Budd-Chiari syndrome caused by a zygomycete. A new pathogenesis of hepatic vein thrombosis.. Arch Pathol Lab Med 113: 1171-1174, 1989.

7. Kurt M, Shorbagi A, Altundag K, Elkiran T, Güllü I, Kansu E. Possible association between Budd-Chiari Syndrome and gemtuzu- mab ozogamicin treatment in a patient with refractory acute myelogenous leukemia. Am J Hematol 80: 213-215, 2005.

8. Mamone G, DiPiazza A, Gentile G, et al. Imaging of calcified hepatic lesions: spectrum of diseases. Abdom Radiol (NY) 1-16, 2021.

9. Waheed N, Cheema HA, Suleman H, Mushtaq I, Fayyaz Z, Anjum N. Is Hepatovenocaval Syndrome a Different Entity from Budd-Chiari Syndrome in Children?. J Coll Physicians Surg Pak 28: 344-347, 2018.

10. Sarawagi R, Keshava SN, Surendrababu NR, Zachariah UG, Eapen EC. Budd-Chiari syndrome complicating hydatid cyst of the liver managed by venoplasty and stenting. Cardiovasc Intervent Radiol 34 (Suppl 2): S202-S205, 2011.

11. Bedioui H, Nouira K, Ayadi S, et al. Budd-Chiari syndrome secondary to hepatic echinococcosis. Gastroenterol Clin Biol 31 (8-9 Pt 1): 721-724, 2007.

12. Mamone G, Cortis K, Sarah A, Caruso S, Miraglia R. Hepatic morphology abnormalities: beyond cirrhosis. Abdom Radiol (NY) 43: 1612-1626, 2018.

13. Hayashi $Y$, Inagaki $K$, Hirota $S$, Yoshikawa $T$, Ikawa H. Epithelioid hemangioendothelioma with marked liver deformity and secondary Budd-Chiari syndrome: pathological and radiological correlation. Pathol Int 49: 547-552, 1999.

14. Okuda K, Kage M, Shrestha SM. Proposal of a new nomenclature for Budd-Chiari syndrome: hepatic vein thrombosis versus thrombosis of the inferior vena cava at its hepatic portion. Hepatology 28: 1191-1198, 1998.

15. Zanetto A, Pellone M, Senzolo M. Milestones in the discovery of Budd-Chiari syndrome. Liver Int 39: 1180-1185, 2019.

16. ShresthaSM, KageM, LeeBB. Hepatic vena cava syndrome: New concept of pathogenesis. Hepatol Res 47: 603-615, 2017.

17. Kucukay F, Akdogan M, Bostanci EB, Ulus AT, Kucukay MB. Percutaneous Transluminal Angioplasty for Complete Membranous Obstruction of Suprahepatic Inferior Vena Cava: Long-Term Results. Cardiovasc Intervent Radiol 39: 1392-1399, 2016.

18. Wang Q, Li K, He C, et al. Angioplasty with versus without routine stent placement for Budd-Chiari syndrome: a randomised controlled trial. Lancet Gastroenterol Hepatol 4: 686-697, 2019.

19. Valla DC. Budd-Chiari syndrome/hepatic venous outflow tract obstruction. Hepatol Int 12 (Suppl 1): 168-180, 2018.

The Internal Medicine is an Open Access journal distributed under the Creative Commons Attribution-NonCommercial-NoDerivatives 4.0 International License. To view the details of this license, please visit (https://creativecommons.org/licenses/ by-nc-nd/4.0/).

(C) The Japanese Society of Internal Medicine Intern Med Advance Publication 